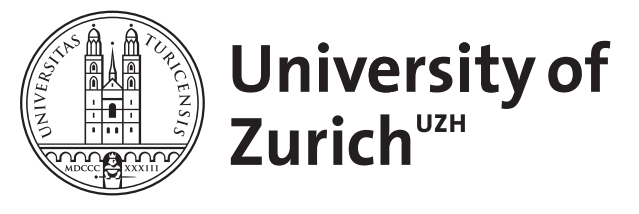

\title{
Architecting play
}

\author{
Franinovic, Karmen
}

\begin{abstract}
From the grotesque pavilions hidden in sixteenth century Italian gardens to the temporary structures in public space in the 70s and recent digitally augmented environments, architectures of play have long been designed to engage explorative experiences. The uncertainty of play allows us to probe new behaviors, to poke into the boundaries of subjectivity and to interact with people, things and systems in unexpected and unfamiliar ways. In this essay, we explore how an interactive system, situated in public space, may foster explorative activities and enable the transformative power of play. Through the analysis of several computer-augmented architectures, we examine what interactive qualities might enhance the transformative power of play. Beginning with the Fun Palace, an early attempt to use cybernetics in order to encourage visitors to experiment with their habits, we move to contemporary projects including some of the author's own work. The latter takes the advantage of existing public locations in order to insert exploratory play into everyday life. Through analysis of these projects, we discuss whether such environments raise our responsibility toward others within our surroundings; whether they enable us to transform our own established behaviors; and whether they empower us to reclaim public space
\end{abstract}

DOI: https://doi.org/10.1007/s00146-010-0292-4

Posted at the Zurich Open Repository and Archive, University of Zurich ZORA URL: https://doi.org/10.5167/uzh-156032

Journal Article

Published Version

Originally published at:

Franinovic, Karmen (2011). Architecting play. AI Society, 26(2):129-136.

DOI: https://doi.org/10.1007/s00146-010-0292-4 


\title{
Architecting play
}

\author{
Karmen Franinovic
}

Received: 14 May 2008/Accepted: 13 August 2010/Published online: 14 September 2010

(C) Springer-Verlag London Limited 2010

\begin{abstract}
From the grotesque pavilions hidden in sixteenth century Italian gardens to the temporary structures in public space in the 70s and recent digitally augmented environments, architectures of play have long been designed to engage explorative experiences. The uncertainty of play allows us to probe new behaviors, to poke into the boundaries of subjectivity and to interact with people, things and systems in unexpected and unfamiliar ways. In this essay, we explore how an interactive system, situated in public space, may foster explorative activities and enable the transformative power of play. Through the analysis of several computeraugmented architectures, we examine what interactive qualities might enhance the transformative power of play. Beginning with the Fun Palace, an early attempt to use cybernetics in order to encourage visitors to experiment with their habits, we move to contemporary projects including some of the author's own work. The latter takes the advantage of existing public locations in order to insert exploratory play into everyday life. Through analysis of these projects, we discuss whether such environments raise our responsibility toward others within our surroundings; whether they enable us to transform our own established behaviors; and whether they empower us to reclaim public space.
\end{abstract}

Keywords Play - Interaction - Responsive architecture . Physicality $\cdot$ Public behavior

K. Franinovic $(\square)$

Zurich University of the Arts,

Interaction Design, Zurich, Switzerland

e-mail: karmen.franinovic@zhdk.ch

URL: http://www.iad.zhdk.ch

K. Franinovic

Zero-Th Association, Zurich, Switzerland

URL: http://www.zero-th.org

\section{Introduction}

\subsection{Constraints of public space}

To design within public space is to design within the space of tensions, such as those between fixed architectural elements and transient urban flows, or those between different groups encountering each others in public. These tensions are reduced and sometimes rendered invisible through careful urban design, concealed commercial strategies, social rules and techniques for the control of public behavior. Shaped by the latter, we often perform automatically_our physical and social actions are based on common-sense practices and learned behaviors. We conduct ourselves according to different contexts: in the office or in the bar we change our social roles (Goffman 1959). Our physical gestures, such as walking, sitting, or shaking hands, express the fashion of the time or identify us as part of a specific social group (Mauss 1934).

These embodiments of behavioral norms are rarely challenged, especially when exposed to public eye. Rather, they are reinforced through the design of public areas. An example is Muzak, a background sound design for commercial areas. It is based on the idea that certain social groups identify with a particular style of music. The background sounds have proven to be capable of shaping human behavior: specific musical content attracts certain consumers, while reducing the loitering of other, undesired visitors. Such subtle techniques sonically preselect the people inside and around commercial areas and extend them beyond their physical limits. Obviously, their purpose is to accommodate the private interests of shop owners rather than to enrich public life (Sterne 2006). Easier to perceive are the "hard constraints" set by architectural elements and urban infrastructure. Buildings, parks, and 
transportation systems frame and shape the transient flows of humans and machines. Such management of movement, established though urban design and planning, directly affects human encounters and the use of public space. A well-known example is the proliferation of highways and suburbs in North America, where urban developers and car industry joined forces to produce profit without considering and designing for pedestrian life.

American highways, as well as Muzak, reduce the chances of encounters between strangers of different backgrounds. These hard and soft systems, seamlessly embedded in our lives since childhood, support the nonegalitarian uses of urban space. They encourage us to keep our distance from the people with whom we share the world. Could playful activities challenge such behavioral patterns?

\subsection{Transformative power of play}

Sports, music, cinema, and other entrainment industries offer play as an activity based on clichéd modes of competitiveness and adventure. Play is sold and controlled because, as theater director Victor Turner argues, it has the power to challenge the established structures: "Playfulness is a volatile, sometimes dangerously explosive essence, which cultural institutions seek to bottle or contain in the vials of games of competition, chance and strength, in modes of simulation such as theater, and in controlled disorientation, from roller coasters to dervish dancing" (Turner 1983). These control mechanisms are the result of the fear caused by one of the most interesting qualities of play: its transformative power.

As much as it can be a social and cultural phenomenon, transformation though play can also be an empowering personal experience. Theater director Eugenio Barba described play as an unpredictable strategy for personal exploration: "The body is deconstructed so that it can be re-created according to the plan. Plans are not fixed. They change, sometimes slowly, sometimes suddenly" (Barba 1986). It takes bravery to embark on such an unpredictable and transformative experience. Yet play is often not taken seriously. After all, it is only play: a temporary event that one can leave at any time (Huizinga 1986). But the experiences of play may be so powerful as to transfer and to extend into everyday life. Dostoyevsky described this invasion of play into the quotidian in his novel The Gambler (sometimes translated as The Player). The main character subordinates himself to a particular kind of play_-gambling — and feels its effects on every aspect of his life. Immersed in play, he cannot return to his daily activities.

If play can so pervasively invade our lives, than it offers an opportunity for personal and collective transformation.
However, one requires great prudence in selecting which kind of play one wants to engage with. The qualities of the game encourage certain types of behaviors: playing a computer game or playing basketball stimulates different kinds of skills and emotions. Considering the variety of game and play potential, how can we stimulate exploration that allows us to question and to transform our own personal and social behaviors? And what is the role of the architecture and the environment in which such exploration takes place?

\section{Designing an architecture of play}

The architectures of play are not composed of the physical building alone, but also entail the flows of light and sound. In the case of interactive environments, those tangible and ephemeral structures are affected by the software system. Most importantly, social interaction is an essential part of the overall ecology of play: an architecture of play cannot exist without the people who activate it. The relations between players, which are created in interaction with their surroundings and constantly transforming, can hardly be predicted or interpreted through computing. Yet many creators of playful environments have attempted to do so.

\subsection{Automaticity: delegating action to architecture}

Joan Littlewood, a British theater director, created works that aimed to develop new forms of social and political expression. However, she was unsatisfied by the limitations of theatrical productions and the ways in which these engaged the participation of the audience. Littlewood dreamed of creating a public architecture that could accomplish similar goals to those she had in her theater works: to enable visitors' active participation without the engagement of a performer and to engage in critical explorations of the quotidian through play. The result of these ideas was the Fun Palace, an architecture of play that would be based on an interactive system open to accident and founded on evolutionary processes whose goal is unknown (Littlewood 1964).

Littlewood invited architect Cedric Price to design a building that would encourage playful activities for its inhabitants. His architectural proposal had no fixed form or plan (Banham et al. 1969). It was a flexible frame with movable modules: smaller cells that hosted cooking and washing services and large volumes such as auditoriums. The functional juxtapositions, Price believed, would create new types of social activities. Rooms, walls, and walkways were to be automatically re-arranged, and resources such as sound, light, temperature, and humidity would be modified to create different atmospheres. Such spatial transformations 
were to have been based on visitors' desires and enabled by a cybernetic system. Price and Littlewood had invited cybernetician Gordon Pask to program an environment that could adapt to the needs of its inhabitants and engage their participation. He proposed the retrieval of information about visitors' needs based on the incessant tracking of their activities (type, size, location, quality, quantity) through electronic sensors and other monitoring devices. Based on the gathered information, the cybernetic system would compare people arriving to those leaving the building, causing it to change its shape.

Pask designed the system based on his idea that architecture must serve and, at the same time, control its inhabitants' behavior (Mathews 2007). In contrast, Littlewood's original plan was to raise visitors' awareness about the constraints through which traditional architecture and social norms shaped their activities. Her idea was to create an environment which would allow for the reflection and change that once relied on professional services such as psychoanalysis: "therapy for everyone: men and women from factories, shops and offices, bored of their daily routine, will be able to re-enact incidents from their own experience in burlesque and mime and gossip, so that they no longer accept passively whatever happens to them, but wake to a critical awareness of reality" (Littlewood and Price 1968). Littlewood valued discussion and antagonistic interactions and stated that the Fun Palace "must provide the creative conflict about ideas that can replace mass murder" (Littlewood and Price 1968). Pask, on the other hand, hoped to avoid any kind of conflict by relying on an automatic decision-making system (Pask 1969). For example, if an equal number of people wanted to perform different activities, the system would try to accommodate both by creating two functional spaces. As the core of the project became the mathematical prediction models applied to social and psychological dynamics, the Fun Palace appeared to change from an exploratory toy to a control machine of an automated society.

The automaticity of cybernetic system might have been the main reason for this change. All the architectural transformations were automatic and based on the input data collected through surveillance mechanisms. The system allowed for minimal direct interaction from the visitors. The only directly addressable interface was the Pillar of Information, proposed by artist Roy Ascott, with which visitors could perform inquiries and through which the system could learn more about visitors' interests. ${ }^{1}$ By preventing voluntary interaction, design choices conflicted with Littlewood's original plan of fostering participation

\footnotetext{
${ }^{1}$ Personal discussion with Roy Ascott in Vienna at Consciousness Reframed: The Planetary Collegium's IXth International Research Conference in 2008.
}

and transformative behaviors. The active engagement of visitors was substituted with the intelligence of an artificial system. Yet both Price and Littlewood never abandoned the idea of play as a strategy to reclaim public agency-Later Price said, "What do we have architecture for? It's a way of imposing order or establishing a belief, and that is the cause of religion to some extent. Architecture does not need those roles anymore... Creating a continuous dialogue with each other is very interesting; it might be the only reason for architecture" (Price 2003).

\subsection{Complexity: location and timing of media responses}

As in the Fun Palace, many contemporary projects capture visitors' actions without their permission and react responsively without the visitors willing engagement. For example, in Camille Utterbach's piece Untitled 6, simply stepping into the installation triggers changes of the video image (Utterbach 2007). Although the feedback to their movement is direct and immediate, visitors do not necessarily make a decision to act: their every movement triggers the response of the environment. This is sometimes the result of the artist's desire to create an immersive experience and is often due to the computer's difficulty in recognizing the participants' intention. In video-tracking systems, human intention is often oversimplified as the quantity of movement: if people move more, their will to act is inferred to be stronger. This limits the potential and richness of participants' interaction with the environment and the people in it (Franinovic 2005).

An alternative to such simplified feedback loops are complex systems designed for responsive media spaces (Sha 2002). The latter combine software evolution models with interfaces embedded in visitors' garments and their surroundings. The algorithms transform the media environment on different time scales: the long responses related to the overall evolution of the space, its temporal behavioral state responses, and the short-time response to individual actions. Similarly to original Fun Palace concept, these architectures are designed "to evolve without a specific goal and to encourage a multiplicity of interactions and behaviors" (Kuzmanovic and Gaffney 2005) (Fig. 1).

The txOom environment (FoAM 2003) is the second in the series of three projects originating in the T-garden research that explored such evolutionary behavior of play spaces (Sha 2005). In it, the participant's movements affected the sonic and light atmosphere as well as the material boundaries composed of FoAM, lycra, electroluminescent wire, and sand. Tangible and wearable interfaces distributed the loci of activity throughout the space. Because they required less direct attention than, for example, a video screen, a stronger sense of immersion was 


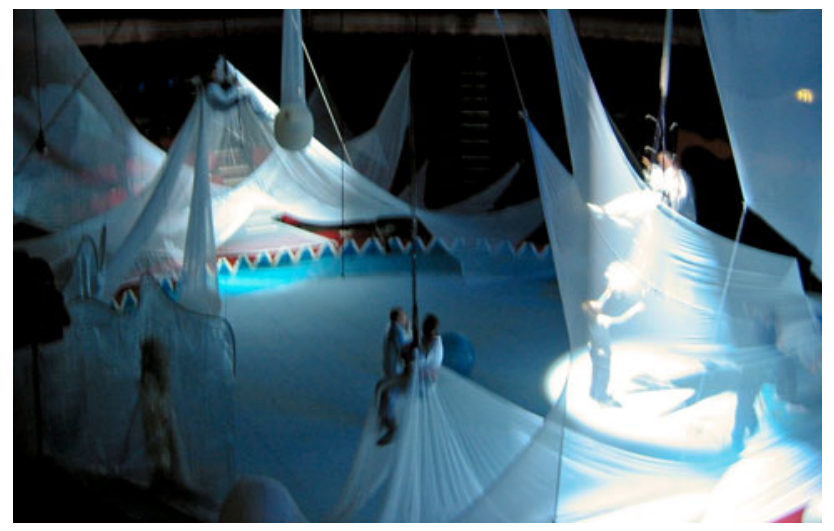

Fig. 1 txOom (FoAM 2003) responsive environment in Hippodrome in Great Yarmouth

created. Rather than interacting with the fixed points of control and display in space, participants could intuit fields of activity and spatially engage with them using their whole bodies. Although most of the media response was global, the individual gesture performed with the costumeinterface was directly coupled to the video projected on the floor. This response in immediate proximity of the player was easily perceivable and highly engaging: as one was moving within the projected image of cloud or smoke, one could send waves toward others in the space. ${ }^{2}$

Shaping the sound, becoming tangled in textiles or swinging in the air, participants became a part of a media ecology in which everything seemed to be connected to everything else. Immersed in rich sonic and luminous dynamics, they sometimes had difficulties in identifying the results of their own actions. One of the participants said, "...never was I aware of altering the environment by my own actions, which was a pity" (Boxer 2002). The rich media feedback seemed not to allow participants to distinguish between the effects of their actions and those of other participants on the changing environment. This often resulted in repetitive bodily movements, as if participants wanted to affirm their intention to perform a certain action and reveal its effects on their surrounding. The voluntary interaction appeared to be blocked by the lack of transparency in coupling participant's action to media response and the complexity of the behavior of the environment.

While simple mappings between the quantity of motion and media feedback allow for an easy understanding of the effects of one's actions (as is the case in Utterbach's work), complex systems risk confusing visitors, who may not be able to perceive a response to their actions in the myriad of environmental changes. The latter are often interpreted as automatic behavior on the part of the environment, because

\footnotetext{
${ }^{2}$ Author's personal experience of txOom installation at Biennale internazionale di Arte Giovane in Turin, Italy in 2002.
}

those changes cannot be perceived as linked to participants' activities. As a result, participants do not feel aware of and responsible for the transformation of their surroundings. Rather, they are mesmerized by its evolution, which appears to be independent from their actions.

\subsection{Tangibility and scale: architectural politeness}

Richard Schechner argued that the sensation of looseness in play emerges though activities rather than from physical structure of the environment (Schechner 1993). The coupling between actions performed and media feedback enables participants to become aware of the influence of their behavior on the environment. However, the physicality of the interface and the control over it plays an important role as well. In txOom, immersive media and soft, comfortable materials helped participants to relax. Participants, however, felt constrained when wearing certain costumes, which was also the intention of the designers. Some of them felt that the situation was out of their control: when hung in the air by bungee cords, one had to rely on the person pulling one up and down, which required a great amount of trust. ${ }^{3}$ The incapability to autonomously act, and the dependency on txOom performers who managed the bungee cords, removed, to a certain extent, the visitors' responsibility for their behavior.

The Hinge Dimension project (Franinovic and Wilson 2007) explored how the physical structure of the environment affects the transparency of its responsive behavior. The goal was to enable citizens to voluntarily change the physical and media architecture of a public location. A church was filled with large screens that could be moved around a pole acting as a hinge (Fig. 3). A movement of a single screen would result in local and global transformation of sound and video image projected on the wooden ceiling of the church. Participants could arrange their surroundings in a labyrinthine and chaotic fashion, or organize it in clusters or corridors. The modification of the overall structure influenced sonic and light composition, which reflected the changing potential of the space for the flow of people in the location (clustered, directional, diagonal structure...) (Fig. 2).

The Hinge Dimension environment responded only when visitors physically grasped its architectural elements. Without the intention to transform the spatial structure, nothing happened, except the resonance of the past activity, which slowly faded into the background. The direct link between visitors' actions upon the architectural elements and the resulting changes in the sonic and luminous atmosphere facilitated their understanding of the system. Satisfied with the immediate response, some participants

\footnotetext{
${ }^{3}$ Personal discussion with one of the txOom visitors, Zurich, 2006.
} 

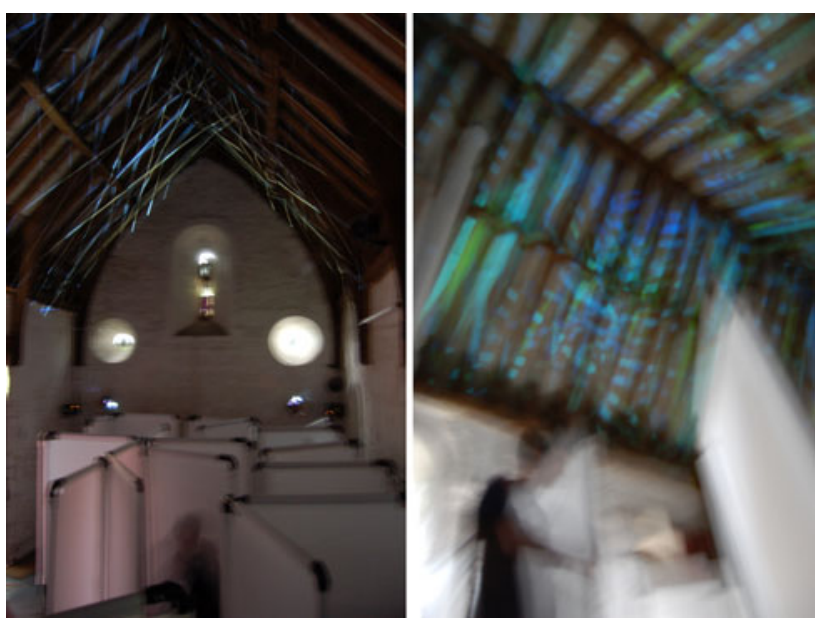

Fig. 2 Hinge Dimension (Zero-Th 2007) at Enter Unknown Territories festival in Cambridge, UK. From the inside of the labyrinth, participants can sense its changing structure through sonic and visual responses of the environment

quickly abandoned the installation. Others stayed longer and discovered the evolving behaviors of the space. However, in no case was the link between oneself, the response of the environment and other people in the space lost. The physicality of interface allowed for such directness and facilitated awareness while maintaining the complexity and richness of an evolving system.

In contrast to the soft materials used in txOom, the atmosphere generated through Hinge Dimension did not provide a cozy setting. The white-walled labyrinth, enclosed in cold stone architecture, and the changing behavior of the system provided a rather challenging context. Cold and hard surfaces caused participants to perceive the environment as "scary and moving", "eerie and wicked" or "sinister, innocent and pure at the same time" (Hinge Dimension comment book, in archives of Essex dance 2007). Another participant wrote: "Felt like being underwater in a way. The white cloth of the walls and the church setting gave the feel of a hospital almost. Otherworldly experience". The players cautiously but exploratively moved within the structure, partially due to the austerity of the setting.

What occasionally obstructed the interaction in Hinge Dimension was the scale of the wall-like interfaces. Some participants shied away from interacting with the large physical interfaces of the labyrinth. One visitor wrote "I was very polite to the space until I was immersed and than enjoyed the changing space" (Hinge Dimension comment book, in archives of Essex dance 2007). In discussion, we discovered that she felt responsible, and partially guilty, for changing the public architecture. The austerity of the building contributed to these feelings, as did the scale of the change she was about to create. This experience of "architectural politeness" emerged with the awareness that by transforming a shared environment one affects other cohabitants. Over the time, this sensation vanished and players focused on using the installation to meet strangers, or to confuse others by rearranging the space.

Although, as Schechner argued, the activities of play are essential in creating the sensation of looseness, the physical structure of the environment has an important role as well. Most participants will shy away from interacting if the interface is too large or if it resembles something that humans do not usually engage with such as building's walls. Moreover, when the response from the system is so large that it affects everyone else in the location, participants tend to reduce their actions.

\subsection{Situatedness: blurring the boundaries of play}

Comparing play to ritual and sacred performances, Johan Huizinga argued that the most important characteristic of play is its spatial separation from ordinary life: "A closed space is marked out for it, either materially or ideally, hedged off from the everyday surroundings" (Huizinga 1986). The FoAM group chose to situate the txOom experiment in the closed space of a circus in the small town of Great Yarmouth, because it allowed them to bring a magical txOom world to isolated community groups (Muller 2002). In the rite of passage from everyday to imaginary space, visitors' feet were washed and massaged, and they were dressed in beautifully crafted garments. Relaxed, participants would sink into a dream world, leaving behind their quotidian worries. The outside world of ordinary life and the inside world of magic were strongly separated.

Contrary to Huizinga, Schechner argued that no fixed space-time boundaries should be established in play. He introduced the flexible concept of a net to describe play space: "a porous, flexible gatherer; a three dimensional, dynamic, flow-though container" (Schechner 1993). Hinge Dimension installation had porous boundaries: it allowed the entrance of anyone at any time without preparatory procedures. Visitors would often expect to enter an empty church and be surprised by the world they found inside. This direct link made the entrance less magical than in txOom, but it made the connection to ordinary life outside of the chapel stronger (Fig. 3).

A step further in merging play and everyday life was taken in the Recycled Soundscapes project (Franinovic and Visell 2004), in which a set of sculptures enable participants to play with an architecture of invisible sonic structures, directly in an open urban space. The three sculptural interfaces have no written or verbal explanation, and interaction with the system is consequently a process of discovery (Fig. 4). The large red Beludire interface allows 


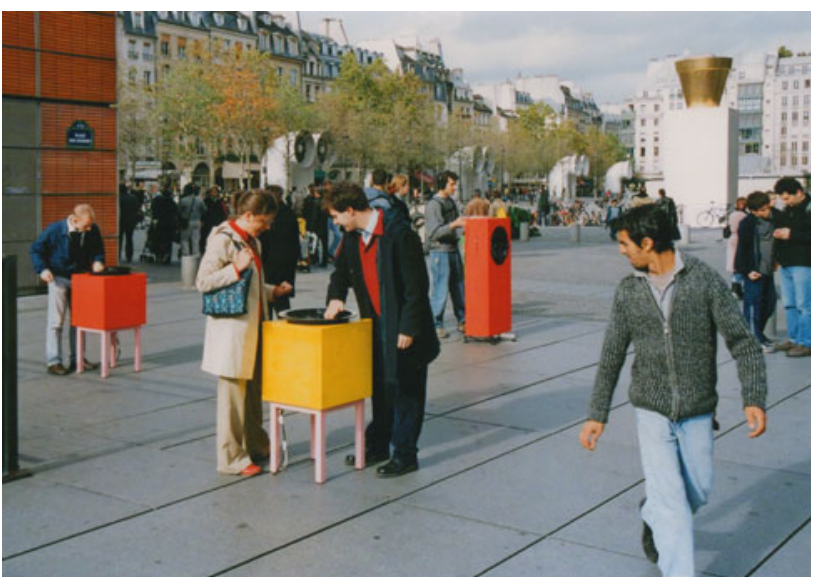

Fig. 3 Recycled Soundscapes (Zero-Th 2004) at Resonances festival 2004 in Paris

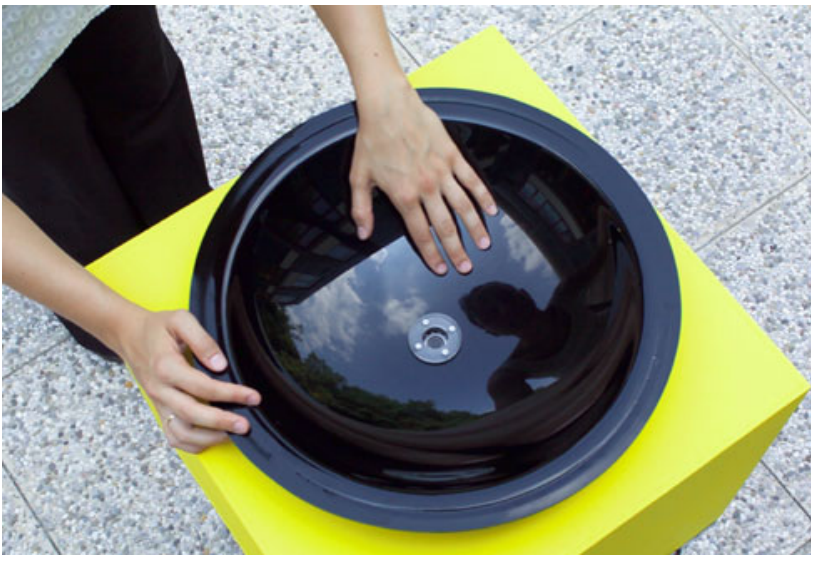

Fig. 4 Sonic Bowl: soundscape collected in the bowl is molded through participant's action

for the listening and recording of distant and transient sounds, which would otherwise be difficult to hear. Once captured, the amplified sounds are echoed following a soft gong sound from loudspeakers integrated within the other two instruments, called Sonic Bowls. The replayed sounds communicate to the player that his or her recordings have been stored in these other sculptures. Sonic Bowls can be used to compose the new soundscape out of recorded sounds. This soundscape is generated through different kinds of spinning of the reflective black dishes, the moving parts of the Sonic Bowls. In this way, one hears and creates a multiplicity of voices molded through one's physical actions (Fig. 4).

The installation forged a connection with existing sonic and social activities (Franinovic and Visell 2007). Some participants recorded their own voices and were eager to find them within the sonic memory of the location accessible through the Sonic Bowls. When listening to their recordings, they might heard a bird, a statement very different from their own, or their own words mixed with other voices. Through this strategy of remixing, the designers' goal was to avoid the use of the system to affirm an established participant's identity (by recording and playing his or her statements), but rather to challenge it. The Beludire opened and extended the sonic as well as the social space of the installation into the existing surroundings. Just as distant ambiances and sound sources were made more proximate, the user of the Beludire felt closer to, and experienced a more intimate relation with, visually distant strangers. Reflexively, the device's conspicuously surveilling nature did not leave many passers-by indifferent to its use. This relation was sometimes understood by the observed individuals as an invitation to communicate and to play, and at other times as an intrusion into their private spheres. Through interaction, the play space continued to shift between centrifugal and centripetal agency. It was capable of becoming for some, a closed and insulating system, and of forging connections to those that were removed from their spatial vicinity for others. The project shows how the porous and flexible boundaries of play, as proposed by Schechner, may be created through an interactive system.

Recycled Soundscapes inserted itself, as a net, into an everyday context. The extension from the installation space into the surrounding area was enabled by the choice of the medium. Sound, as transient and pervasive phenomena, involved all the passers-by in the location-even those who did not have any interest or time to interact. Using the Beludire, players would reach out into the sonic domains of passers-by, including them in play. Questions emerged: "What makes a conversation in an urban area private? What can one do with the transient non-tangible information floating in the public? How close can one come to a stranger?" The use of sounds existing in the location allowed participants to explore and to play with what was already present in their daily life. By playing with what is already there, participants engaged with the existing social relationships and actively tackled questions related to the auditory use of public space.

\section{Conclusions}

The projects presented show that the architectures of play can help us to engage with established behaviors in public space. Unfamiliar physical and social experiences in which we normally would not participate can be encouraged through play. Play often depends on the design of an architecture of play. In this essay, we discussed design decisions that appear to have had the most importance in our own work: choice of location, scale and physicality of 
interface, timing and localization of media response to participant's actions, and automaticity embedded in the system.

Firstly, the way in which an architecture of play is situated within public space has a great effect on connecting the playful experiences with everyday life. When players act within the boundaries of the dream world, such as an amusement park or a casino, they have an opportunity to access completely new experiences. But when participants act within an everyday public setting, they are empowered to self-criticize and challenge their own established behaviors (e.g., Recycled Soundscapes). The awareness of the existing behavioral patterns, and the opportunity to alter them through play, allows for the questioning and transformation of players habits. In this sense, a temporary architecture of play situated in an everyday context can have a greater effect on players' behavior than a permanent one.

Secondly, the scale and physicality of an interface may affect participation and comfort of players (e.g., Hinge Dimension). Habitually, our interaction with the world is on a smaller scale: How many times do we push a button every day? How many times do we install a new window in our flat or rearrange the furniture of our office? Moving to a different home, erecting a new wall or taking it down: tangible changes of our surroundings are associated with the reorganization of our lives. They are often considered stressful, but can also be empowering. Therefore, we must consider the effect that the scale of physical interface may have on its user, and be aware of "architectural politeness" behaviors.

Thirdly, players must be able to differentiate between feedback caused by their voluntary and involuntary interactions. Physicality of interaction can help raise awareness and responsibility for one's actions within an architecture of play, especially in dense media environments. To willingly engage with physical objects is to decide to reach into the world and act upon it. The responsibility increases with tangibility: throwing a physical glass has a very different effect on our surroundings than does throwing a digital image of a glass. Physicality is linked to causality and visibility of one's actions. In an urban setting, our actions are exposed to the public eye and others are affected by our performance (e.g., when recording the voices of others through Beludire). On the one hand, this exposure creates an opportunity to play with public behaviors, but on the other, it makes it harder for passers-by to get involved because they may feel observed and judged.

Finally, the transparent relationship between action and media response allows us to consciously act within an interactive environment. Both the automaticity of interaction (e.g., Fun Palace and Untitled 6 ) and the complexity of the system may lead to one's unawareness of one's actions (e.g., txOom and Hinge Dimension). If the interactive system is based on a question-answer or action-reaction model, the process will result in a problem-solving game. Once the logic of the system is discovered, the players feel as if they have answered the question that is posed and the exploration is over. A non-transparent evolving environment requires an openness and willingness to interact and take risk. An ever-changing surrounding is frightening-its cycles are unknown and its seasons can affect our moods. In complex evolution-based systems and automatic ones, the attention of the participants tends to divert toward observing or ignoring the system respectively rather than engaging with each other.

In conclusion, in order to engage in critical exploration of everyday life, players must have a clear understanding of the effect of their behavior on that of the environment. By raising the awareness of participants' actions, the responsibility shifts from the automatic system to the participants. The interface serves as an instrument of coconstruction of the world, rather than a boundary for the exchange of data. In this context, instead of serving the purpose of the social control machines, technology and architecture may become the enablers of social and personal transformation.

\section{References}

Banham R, Barker P, Price C (1969) Non-plan: an experiment in freedom. New Soc 12(338):435-441

Barba E (1986) Beyond the floating islands. PAJ Publications, New York

Boxer S (2002) txOom: quiet an experience, Great Yarmouth, available at: http://www.futurephysical.org/pages/content/wearable/wearme/ article_txoom_sb.htm. Accessed 1 Aug 2010

FoAM (ed) (2003) [tk's:um]. Kibla, Maribor. Also: http://www.fo.am. Accessed 1 Aug 2010

Franinovic K (2005) Behavioural spaces, designing for the social and sensorial ecologies in public spaces. $\mathrm{n}$ : theatre, dans, etcetera. (96) pp 22-30

Franinovic K, Visell Y (2004) Recycled soundscapes. In: Proceedings of the 5th conference on designing interactive systems: processes, practices, methods, and techniques (Cambridge, MA, USA, August 01-04, 2004). DIS '04. ACM, New York, NY, 317

Franinovic K, Visell Y (2007) New musical interfaces in context: sonic interaction design in the urban setting. In: Proceedings of the 7th international conference on new interfaces for musical expression (New York, NY, June 06-10, 2007). NIME '07. ACM, New York, NY, 191-196

Franinovic K, Wilson A (2007) Hinge dimension: an exploration of enaction in architecture. In: Proceedings of the 4th international conference on enactive interfaces, pp 101-105

Goffman E (1959) The presentation of self in everyday life. Doubleday, New York

Hinge dimension comment book, in archives of essex dance (2007) Cambridge, UK. Available at http://zero-th.org/HingeDimension. html. Accessed 1 Aug 2010

Huizinga J (1986) Homo ludens: a study of the play-element in culture. Beacon Press, New York 
Kuzmanovic M, Gaffney N (2005) Human-scale systems in responsive environments. IEEE Multimed 12(1):8-13

Littlewood J (1964) Fun palace: promotional brochure. Canadian Centre for Architecture. Available at http://svrdam.cca.qc. ca/ZooMI/Default.aspx?obj=DR1995:0188:001:016. Accessed 24 Aug 2010

Littlewood J, Price C (1968) The fun palace. Drama Rev 12(3):127134

Mathews S (2007) From agit-prop to free space: the architecture of cedric price. Black Dog Publishing, London

Mauss M (1934) The technology of the body. In: Kwinter S , Crary J (eds.) (1992) Incorporations, Zone, New York, pp 455-477

Muller N (2002) Report: txOom public experiment, available at: http://libarynth.org/project_txoom_user_research. Accessed 1 Aug 2010
Pask G (1969) The architectural relevance of cybernetics. AD: architectural design (494-496)

Price C (2003) Re: CP. Birkhäuser, Basel

Schechner R (1993) The future of the ritual: writings on culture and performance. Routledge, New York

Sha XW (2002) Resistance is fertile: gesture and agency in the field of responsive media. Configurations 10(3):439-472

Sha XW (2005) The Tgarden performance research project. Mod Drama 48(3):585-608

Sterne J (2006) Urban media and the politics of sound space. In: OPEN sound: the importance of the auditory in art and the public domain, (9) (Fall 2005) pp 6-15

Turner V (1983) Brain, body and culture. Zygon 18(3):221-245

Utterbach C (2007) Artist website available at: http://www. camilleutterback.com. Accessed 1 Aug 2010 